\title{
Angiogenesis is associated with the onset of hyperplasia in human ductal breast disease
}

\author{
JE Bluff', SR Menakuru', SS Cross², SE Higham', SP Balasubramanian', NJ Brown', MW Reed' and CA Staton*,I \\ 'Microcirculation Research Group, Academic Unit of Surgical Oncology, University of Sheffield Medical School, Sheffield, South Yorkshire, UK; \\ ${ }^{2}$ Academic Unit of Pathology, University of Sheffield Medical School, Sheffield, South Yorkshire, UK
}

BACKGROUND: The precise timing of the angiogenic switch and the role of angiogenesis in the development of breast malignancy is currently unknown.

METHODS: Therefore, the expression of CD3I (pan endothelial cells (ECs)), endoglin (actively proliferating ECs), hypoxia-inducible factor-I (HIF-I $\alpha$ ), vascular endothelial growth factor-A (VEGF) and tissue factor (TF) were quantified in I40 surgical specimens comprising normal human breast, benign and pre-malignant hyperplastic tissue, in situ and invasive breast cancer specimens.

RESULTS: Significant increases in angiogenesis (microvessel density) were observed between normal and benign hyperplastic breast tissue $(P<0.005)$, and between in situ and invasive carcinomas $(P<0.0005)$. In addition, significant increases in proliferating ECs were observed in benign hyperplastic breast compared with normal breast $(P<0.05)$ cancers and in invasive compared with in situ cancers $(P<0.005)$. Hypoxia-inducible factor- $\mid \alpha$, VEGF and TF expression were significantly associated with increases in both angiogenesis and proliferating ECs $(P<0.05)$. Moreover, HIF- I $\alpha$ was expressed by $60-75 \%$ of the hyperplastic lesions, and a significant association was observed between VEGF and TF in ECs $(P<0.005)$ and invasive tumour cells $(P<0.01)$.

CONCLUSIONS: These findings are the first to suggest that the angiogenic switch, associated with increases in $H I F-I \alpha, V E G F$ and TF expression, occurs at the onset of hyperplasia in the mammary duct, although the greatest increase in angiogenesis occurs with the development of invasion.

British Journal of Cancer (2009) I 01, 666-672. doi:10.1038/sj.bjc.6605196 www.bjcancer.com

Published online 21 July 2009

(C) 2009 Cancer Research UK

Keywords: angiogenesis; VEGF; tissue factor; HIF- $\alpha$; breast cancer; hyperplasia

Breast cancer is the most frequently diagnosed cancer in women in both Europe and the United States, with an estimated 608380 new cases of invasive disease in 2007 (Ferlay et al, 2007). Breast cancer is a genetically heterogeneous disease, and in contrast to the clearly defined pathway from normal through adenomatous to invasive malignancy associated with increasing dysplasia in colorectal cancer, there is considerable uncertainty about the pathogenesis of different forms of breast cancer and the relationship between hyperplasia and carcinoma of the breast (Wiechmann \& Kuerer, 2008). However, there is good evidence of an increased risk of breast cancer associated with some hyperplastic lesions (for example, atypical ductal hyperplasia; ADH) and clear evidence of progression from in situ to invasive disease, particularly in highnuclear-grade lesions, although at a relatively low rate, for example, $5-10 \%$ over 10 years (Jones, 2006).

Many cancer patients display a hypercoagulable state leading to complications, such as deep vein thrombosis and pulmonary embolism (reviewed by Bluff et al, 2008). Upregulation of tissue factor (TF), the primary initiator of the coagulation cascade, has a

*Correspondence: Dr CA Staton, Microcirculation Research Group, Academic Unit of Surgical Oncology, School of Medicine and Biomedical Sciences, University of Sheffield, Beech Hill Road, Sheffield, South Yorkshire SIO 2RX, UK. E-mail: c.a.staton@sheffield.ac.uk

Received 17 April 2009; revised 10 June 2009; accepted 30 June 2009; published online 21 July 2009 key role in this process (Molnar et al, 2007). In normal physiological conditions, TF, a $47 \mathrm{kDa}$ transmembrane protein, is constitutively expressed by subendothelial cells (such as pericytes, smooth muscle cells and fibroblasts), whereas vascular endothelial cells (ECs) and intravascular cells do not express TF (Abe et al, 1999). Initiation of the extrinsic coagulation pathway occurs when $\mathrm{TF}$ is exposed to the bloodstream, either after damage to the normal integrity of the vascular EC lining or on activation of monocytes or ECs. Factor VIIa (FVIIa) then binds to TF on the cell surface. Sequential downstream activation of haemostatic protease complexes leads to the generation of thrombin, with subsequent platelet activation and the formation of a fibrin clot, which restores vessel integrity (reviewed by Rak et al, 2006).

During tumourigenesis, the strict regulation of TF expression is lost. Upregulation of TF protein by tumour cells and associated stromal cells has been well documented in breast cancer and other malignant tumours, and may contribute to tumour angiogenesis, metastasis, hypercoagulability and tumour cell survival (reviewed by Bluff et al, 2008). Furthermore, correlations of elevated TF expression with advanced stages of malignancy have been reported in different cancers, including colon (Nakasaki et al, 2002) and breast (Ueno et al, 2000), suggesting that TF may have a role in tumour progression. In addition, TF is known to contribute to tumour progression indirectly, through the role in haemostasis, and, in part, by upregulating the pro-angiogenic vascular endothelial growth factor-A (VEGF) (Zhang et al, 1994; Abe 
et al, 1999). VEGF, a $34-45 \mathrm{kDa}$ protein, is the most potent known stimulator of angiogenesis (the outgrowth of new capillaries from an existing vascular bed), an essential process in tumour growth, progression and metastasis (Folkman, 1995). Although TF has the potential to induce VEGF expression, the primary driving force for angiogenesis is oxygen concentration, and VEGF is known to be upregulated by hypoxia-inducible factor-1 (HIF-1 $\alpha$ ), in regions of hypoxia within solid tumours (Mizukami et al, 2007).

The stage at which angiogenesis occurs in tumour progression is known as the 'angiogenic switch' (Folkman et al, 1989). Angiogenesis in invasive breast cancer is well documented (Bos et al, 2005), but relatively few studies have addressed the role of angiogenesis in pre-malignant ductal disease or where the angiogenic switch occurs during the development of breast malignancy. This study therefore quantifies angiogenesis and the number of proliferating ECs in addition to the expression of HIF$1 \alpha$, VEGF and TF in hyperplastic lesions, in situ and invasive breast carcinomas, to determine whether angiogenesis may have a role in dysplastic transformation.

\section{MATERIALS AND METHODS}

\section{Patients}

Archival histological specimens were obtained from 140 patients at the Royal Hallamshire Hospital between 1995 and 2003 with approval from the Local Research Ethics Committee (Ethics number SSREC 98/137). Haematoxylin and eosin sections of all specimens were reviewed by SSC (a consultant histopathologist) and these consisted of (a) normal breast tissue from patients undergoing breast reduction surgery $(n=8)$; (b) benign hyperplastic breast tissue (usual ductal hyperplasia; UDH $(n=30)$ ); (c) ADH $(n=27)$; (d) in situ cancer (ductal carcinoma in situ (DCIS); low/intermediate nuclear grade $(n=19))$; (e) highnuclear-grade DCIS $(n=25)$; and (f) invasive breast cancer specimens (invasive ductal carcinoma; IDC; $(n=31)$ ). Breast cancer patients were excluded from the study if they received neo-adjuvant treatment.

\section{Immunohistochemistry}

Formalin-fixed ( $10 \%$ formaldehyde), paraffin-embedded breast specimens were sectioned $(5 \mu \mathrm{m})$ and mounted on 3-aminopropyltriethoxysilane (Sigma Aldrich, Dorset, UK)-coated slides. Immunohistochemistry was performed, at working concentrations of $10-25 \mu \mathrm{g} \mathrm{ml}^{-1}$, using a panel of antibodies to human platelet EC adhesion molecule-1 (PECAM-1/CD31; $20 \mu \mathrm{g} \mathrm{ml}^{-1}$ ) (M0823; Dakocytomation Ltd, Cambridgeshire, UK), endoglin (CD105; $25 \mu \mathrm{g} \mathrm{ml}^{-1}$ ) (M3527; Dakocytomation Ltd), HIF-1 $\alpha$ (610959 BD Pharmingen, Oxford, UK; $20 \mu \mathrm{g} \mathrm{ml}^{-1}$ ), VEGF (A-20; Santa Cruz Biotechnology, Wiltshire, UK; $10 \mu \mathrm{g} \mathrm{ml}^{-1}$ ) and TF (4509; America Diagnostica Inc, CT, USA; $20 \mu \mathrm{g} \mathrm{ml}^{-1}$ ). A standard horseradish peroxidase staining procedure was followed using an appropriate biotinylated secondary antibody (Vector laboratories, Peterborough, UK) at $5-10 \mu \mathrm{g} \mathrm{ml}^{-1}$, followed by the elite ABC kit (Avidin: Biotinylated enzyme Complex; Vector laboratories) and $\mathrm{DAB}$ (3, 3'diaminobenzidine) as the chromogen substrate (Vector laboratories). All sections were counterstained with Gill's haematoxylin (Sigma Aldrich) to visualise cell nuclei. For HIF- $1 \alpha$ staining, antigen retrieval was performed by heating sections at $95^{\circ} \mathrm{C}$ for $45 \mathrm{~min}$ in target retrieval solution (Dakocytomation Ltd). For all other antibodies, the same antigen retrieval was performed: sections were heated in a microwave in TRIS/EDTA for $3 \mathrm{~min}$ on high power, followed by $7 \mathrm{~min}$ on low power. Appropriate normal sera and casein, both diluted 1:10 in phosphate-buffered saline, were used for blocking all sections and dilution of antibodies. Samples known to be positive for each factor (wounds and invasive breast cancer tissue) were included in each staining run. Negative controls were achieved by replacement of the primary antibody with mouse $\mathrm{IgG}_{1}$-irrelevant antibodies. Furthermore, specificity of the VEGF antibody was confirmed by pre-absorption with a blocking peptide to VEGF (sc-152P; Santa Cruz Biotechnology). A recombinant TF protein (2339-PA; R \& D Systems, Oxfordshire, UK) was used as a pre-absorption control for TF immunostaining because no blocking peptides were available for this factor.

\section{Microvascular density and proliferating EC assessment}

Microvascular density (MVD), a surrogate marker of angiogenesis, was assessed using the Chalkley grid method, as described previously (Vermeulen et al, 2002). Briefly, the tissue was screened at low magnification $(\times 40)$ for the five most microvascular dense areas within each stained specimen ('vascular hotspots') that were $<150 \mu \mathrm{m}$ from any adjacent lesion. Subsequently, each vascular hotspot was viewed at higher magnification $(\times 400)$ using a Chalkley grid graticule, which has 25 randomly placed dots. Under high power, the dots were aligned to touch the maximum number of vessels and these were counted. This resulted in a Chalkley grid score for each hotspot; the sum of the Chalkey scores for each of the five hotspots was termed the cumulative Chalkley score (CCS). CD31 staining was used to assess MVD; endoglin staining (CD105) was used to assess the number of proliferating ECs in each breast specimen.

\section{Quantification of HIF- $1 \alpha$, VEGF and TF staining}

All analyses were carried out by an assessor fully trained by a consultant histopathologist (SSC). HIF- $1 \alpha$ staining was considered to be positive when localised to the nuclei of $>10 \%$ of ductal epithelial cells (Chen et al, 2005). Pure cytoplasmic staining was considered to be negative. Vascular endothelial growth factor-A staining, if present in a lesion, was observed in $>90 \%$ of cells; this was assessed semi-quantitatively using a grading system, which reflected the intensity of staining present within the specimen $(0=$ no staining; $1=$ weak staining; $2=$ moderate staining; and $3=$ strong staining). In contrast, HIF- $1 \alpha$ and TF staining were graded as present or absent, because no obvious differences in the staining intensity of these factors were observed in the different specimens examined. An invasive breast cancer specimen was regarded as positive for TF expression when staining was identified in $>10 \%$ of the tumour cells in the section.

\section{Intra- and interobserver error}

The reproducibility of the Chalkley grids method for quantifying MVD/proliferating ECs, HIF-1 $\alpha$, VEGF and TF staining was established by at least $10 \%$ of the slides being counted by a second experienced observer in a blinded manner. A selection of the slides was also counted for a second time by the first observer. Intra- and interobserver error was assessed 4 weeks apart, on 20 slides from each immunohistochemically stained group and analysed statistically.

\section{Follow-up}

Follow-up information, including the date and cause of death, were gathered by written enquires to the Trent Cancer Registry, according to the ethics committee approval. Patients who had died during the follow-up period from cancer-unrelated causes were treated as censored by statistical survival analysis.

\section{Statistical analysis}

Statistical analysis was conducted using SPSS v15 software (SPSS Inc. Chicago, IL, USA). Appropriate non-parametric tests were 

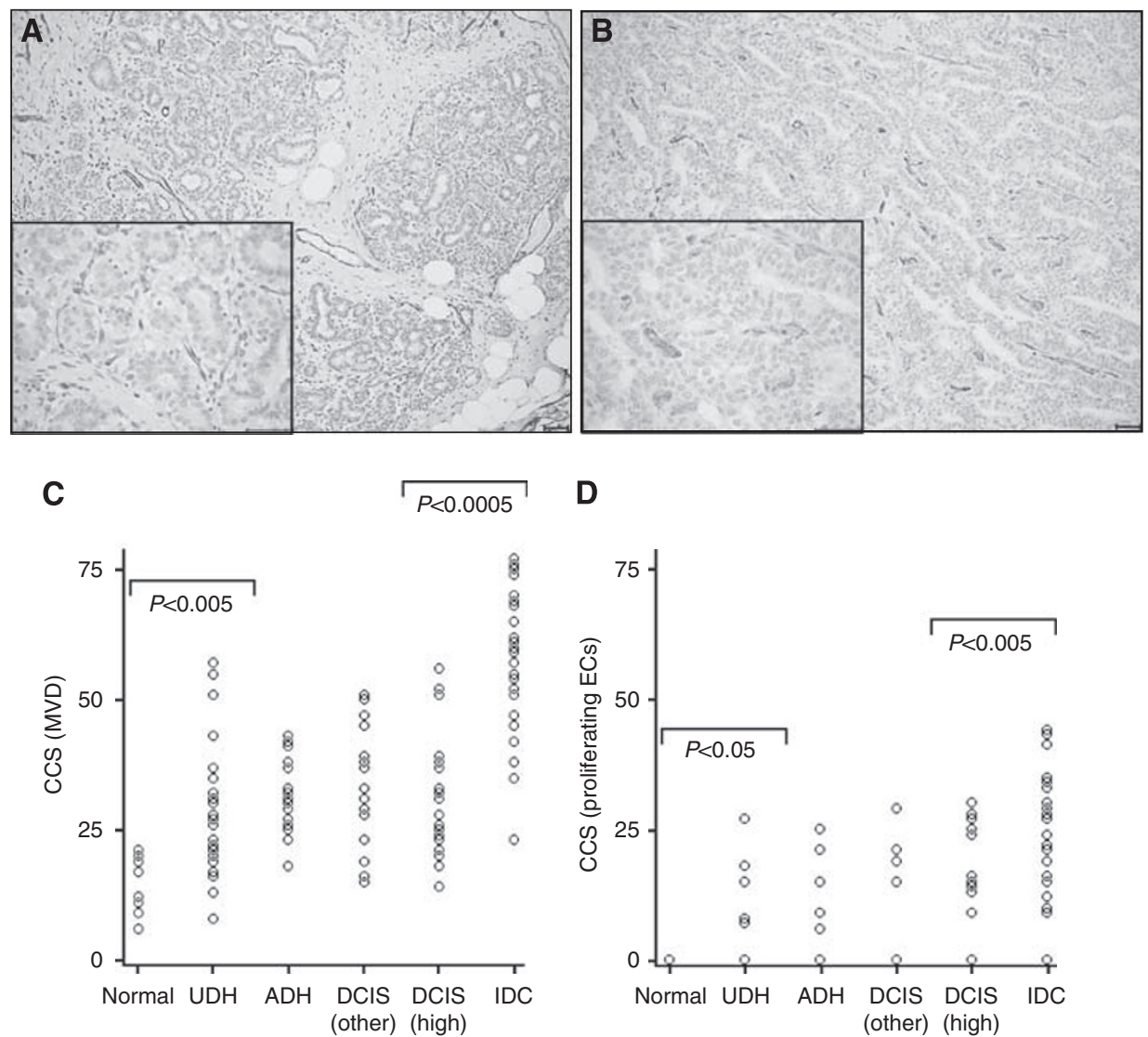

D

Figure I Endothelial staining in breast lesions. Immunohistochemical staining of ECs with (A) CD3I and (B) endoglin in high-grade DCIS. Note the increased frequency and intensity of stained ECs for CD3I compared with endoglin (scale $=50 \mu \mathrm{m})$. Scatter plots representing cumulative Chalkley scores in different breast tissue samples immunostained with (C) CD3I (MVD) and (D) endoglin (proliferating ECs). (C) There was a significant increase in MVD between normal breast tissue and UDH $(P<0.005)$, and a further significant increase in MVD between in situ (DCIS) and invasive breast cancers $(P<0.0005)$. (D) There was a significant increase in proliferating ECs in UDH cases compared with normal breast $(P<0.05)$ and in IDC compared with highgrade DCIS specimens $(P<0.005)$. $P<0.05$ was considered significant (Kruskal-Wallis followed by Mann-Whitney U-test).

used to investigate protein expression (Mann-Whitney U-test for two independent groupings, Kruskal-Wallis test for more than two independent groupings and Jonckheere-Terpstra test for ordinal categorical groupings). If continuous data were not normally distributed, correlations were analysed with Spearman's correlation statistics. Survival curves were plotted using the Cox regression model and analysis was carried out using 'death due to breast cancer' as the end point for overall survival. The influence of MVD and number of proliferating ECs was assessed using the Cox proportional hazards regression model. Data were considered statistically significant at the level of $P<0.05$.

\section{RESULTS}

\section{Assessment of angiogenesis}

Strong CD31 staining was observed along the cell membrane of ECs in all the breast specimens observed (Figure 1A). The median CD31 MVD count was 14.5 in normal breast (range, 6-21; Figure 1C), which increased significantly to 28 in benign hyperplastic tissue (UDH) (range, $13-57 ; P<0.005$ ). Microvascular density scores were constant in UDH/ADH breast samples and in in situ cancers of all grades, but there was a further significant increase in MVD between in situ and invasive carcinomas, with a median MVD count of 31 (range, 14-56) in high-grade DCIS and 59 (range, $23-74)$ in IDC $(P<0.0005)$.
The immunostaining of vessels with CD31 (pan ECs) exhibited an increased frequency and intensity, when compared with endoglin, in all types of breast tissues (Figure $1 \mathrm{~A}$ and $\mathrm{B}$ ). In contrast to CD31 staining, endoglin was not expressed in normal breast ECs, but was expressed by ECs in $17 \%$ of UDH cases, $19 \%$ of ADH cases, $21 \%$ and $44 \%$ of DCIS cases (low/intermediate grade and high grade, respectively) and in $84 \%$ of invasive carcinomas (Figure 1D). Moreover, there was a significant correlation between vessels staining for both endoglin and CD31 (Spearman's rho correlation coefficient $=0.516 ; P<0.005)$. The correlation coefficients for inter- and intraobserver error scores for MVD and proliferating ECs were 0.91 and 0.86 , and 0.92 and 0.89, respectively, showing a high level of agreement.

\section{Assessment of HIF- $1 \alpha$}

Hypoxia-inducible factor- $1 \alpha$ protein was not expressed in normal breast tissue, but was expressed in the nuclei of ductal epithelial cells in $65-75 \%$ of hyperplastic breast/in situ cancers (Figures $2 \mathrm{~A}$ and $3 \mathrm{~A}$ ) and in over $90 \%$ of invasive cancers (Figures $2 \mathrm{~B}$ and $3 \mathrm{~A}$ ). There was a moderate correlation between HIF- $1 \alpha$ expression and MVD (Spearman's rho correlation coefficient $=0.502$, $P=0.001$ ) and proliferating ECs (Spearman's rho correlation coefficient $=0.301, P=0.001)$. The correlation coefficients for inter- and intraobserver error scores for HIF- $1 \alpha$ assessment were 0.88 and 0.91 , respectively, showing a high level of agreement. 

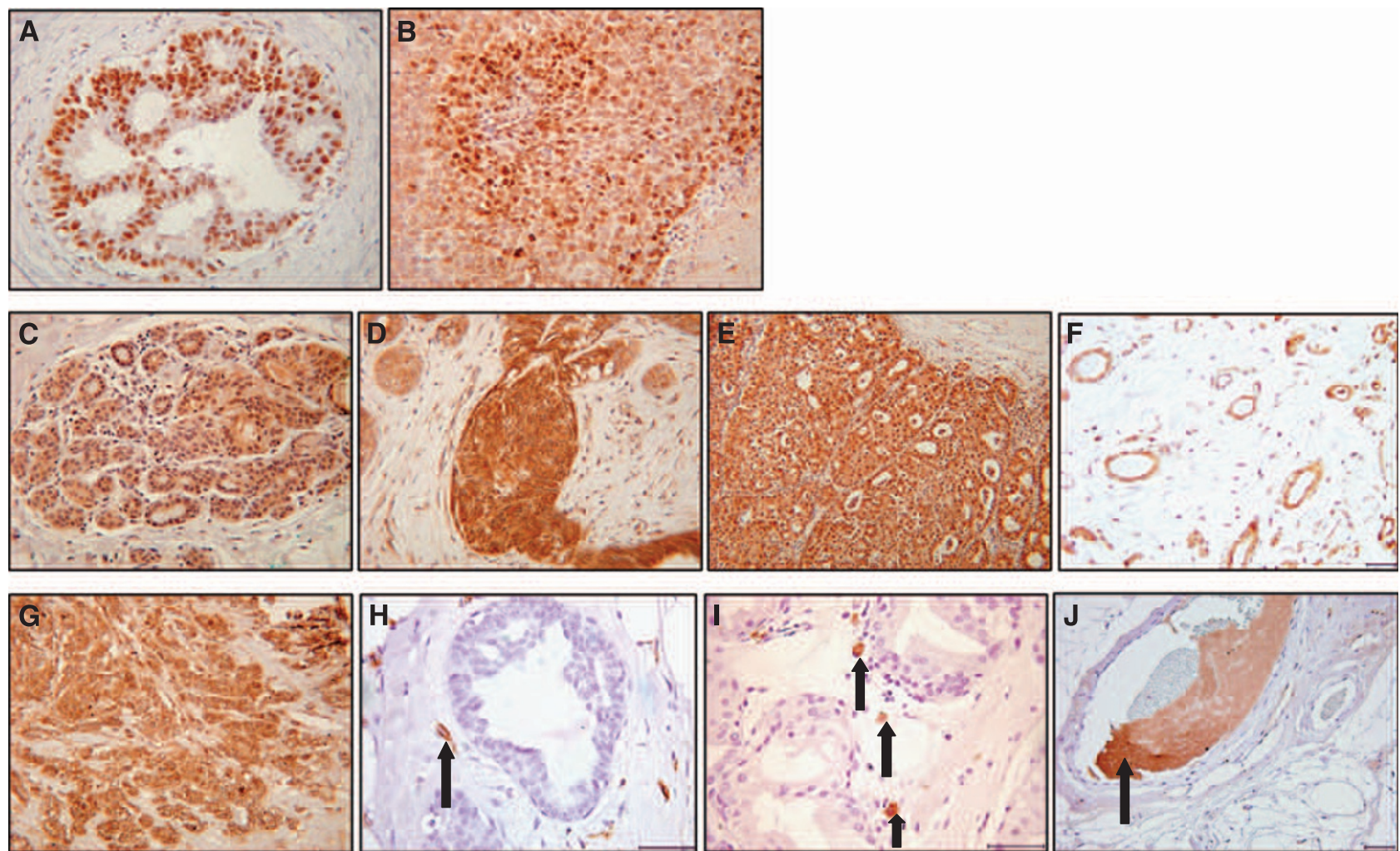

Figure 2 Immunohistochemical staining for HIF- $\mid \alpha$, VEGF and TF. (A) Nuclear HIF- $\mid \alpha$ staining in the ductal epithelial cells of an ADH case and (B) tumour cells of an invasive cancer. (C) Weak expression of VEGF in normal breast epithelium $($ score $=1)$, $(\mathbf{D})$ strong expression in florid usual ductal hyperplasia (score $=2 / 3$ ) and $(\mathbf{E})$ strong staining localised to tumour cells within invasive breast carcinomas (score $=3$ ). $(\mathbf{F})$ VEGF expression in ECs in normal breast tissue. (G) Tumour cells expressed TF in approximately 55\% of invasive breast cancer specimens. (H) TF was expressed in ECs associated with benign hyperplastic tissue (arrow). (I) Putative macrophages expressing TF associated with areas of DCIS (arrows). (J) TF expressed in vessel containing thrombosis (arrow). Photographs $\mathbf{A}-\mathbf{E}$ and $\mathbf{G}$ were taken at $\times 20$ magnification and all others at $\times 40$ magnification.

\section{Assessment of VEGF}

Vascular endothelial growth factor-A was expressed in the cytoplasm of ductal epithelial cells in $100 \%$ of breast specimens, with weak and/or moderate expression in normal breast tissues (Figures 2C and 3B), with moderate to strong expression in hyperplastic tissue/in situ cancers (Figures 2D and 3B) and with strong expression localised to invasive tumour cells (Figures 2E and $3 \mathrm{~B}$ ). Vascular endothelial growth factor-A was also constitutively expressed in breast ECs of normal, hyperplastic and DCIS specimens (Figure 2F), but in contrast to VEGF staining in epithelial cells, there was no change in the EC VEGF intensity in different breast tissue types. Interestingly, most ECs within invasive carcinomas did not express VEGF, whereas stromal cells, such as macrophages and fibroblasts, (based on nuclear and cellular morphology analysis) expressed VEGF in all the different classes of breast tissue examined.

There was a strong correlation between VEGF expression and MVD (Spearman's rho correlation coefficient $=0.731$, $P=0.001$ ) and proliferating ECs (Spearman's rho correlation coefficient $=0.576, P=0.001$ ), and a moderate correlation between VEGF expression and HIF-1 $\alpha$ (Spearman's rho correlation coefficient $=0.317, \quad P=0.001)$ in epithelial/tumour cells. The correlation coefficients for inter- and intraobserver error scores for VEGF assessment were 0.85 and 0.90 , respectively, showing a high level of agreement.

\section{Assessment of TF}

In contrast to the constitutive expression of VEGF in ductal epithelial cells, TF was not expressed in normal/hyperplastic breast epithelial cells or in in situ cancer cells, but interestingly, TF was expressed by tumour cells in approximately $55 \%$ of invasive carcinomas (Figure $2 \mathrm{G}$ ). Tissue factor was not expressed by ECs in normal breast tissue, but was expressed by some ECs in hyperplastic tissue and in ECs associated with in situ cancer (Figure 2H). Similar to the pattern of VEGF staining within the intratumoural vasculature, ECs within invasive cancers rarely expressed TF. Putative macrophages (based on nuclear morphology analysis) expressed TF in all breast tissue types, except normal breast (Figure 2I), and TF expression was also observed in thrombosed vessels within IDC specimens (Figure 2J). The latter were used as an internal positive control for TF immunostaining.

Moderate correlation was seen between TF expression and MVD (Spearman's rho correlation coefficient $=0.431, P=0.001$ ) and proliferating ECs (Spearman's rho correlation coefficient $=0.403$, $P=0.001$ ), and a strong correlation between TF and VEGF expression (Spearman's rho correlation coefficient $=0.593$, $P=0.001)$ in epithelial/tumour cells. In contrast, there was no correlation between TF and HIF- $1 \alpha$ expression in epithelial/ tumour cells. The correlation coefficients for inter- and intraobserver error scores for TF assessment were 0.89 and 0.93 , respectively, showing a high level of agreement.

\section{Survival analysis}

All patients with invasive breast cancer at the time of diagnosis were followed up for a median of 71 months (range, 24-117 months). The Cox regression curve for MVD and proliferating ECs (Figure 4) showed a decreased survival in invasive breast cancer patients exhibiting a high CCS (greater than the median (59 for MVD and 22 for proliferating ECs)) when compared with patients 

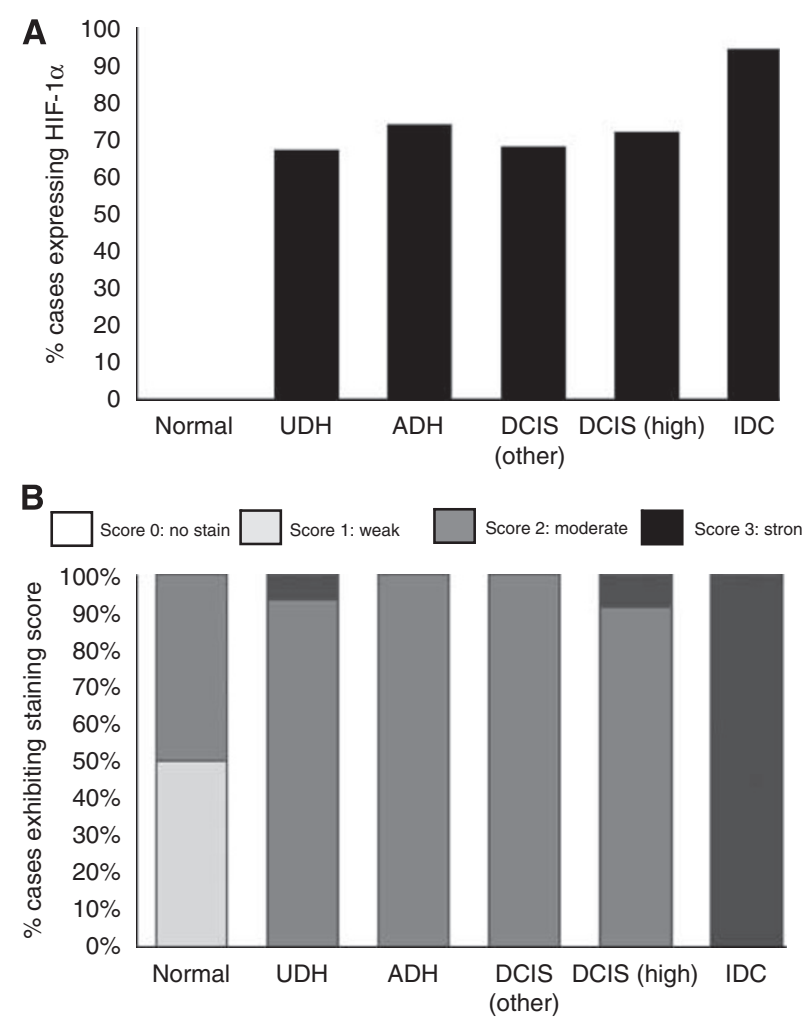

Figure 3 Expression of HIF- $\mid \alpha$ and VEGF in the epithelial/tumour cells of breast lesions. (A) Percentage of cases expressing HIF-I $\alpha$. There is a significant increase in $\mathrm{HIF}-1 \alpha$ expression seen with increasing severity of lesion $(P<0.0$ I) (B) Percentage of cases with varying VEGF expression. There is a significant increase in VEGF expression seen with increasing severity of lesion $(P<0.00 \mathrm{I})$.

with a low CCS (equal to or less than median for both parameters), which did not achieve significance $(P=0.09$ and $P=0.12$, respectively), probably because of the relatively small numbers of patients in the study.

\section{DISCUSSION}

The process of developing a high-density vascular network that connects the tumour and host circulation, termed the 'angiogenic switch,' is a crucial step for the progression of a tumour from a benign to malignant state (Folkman et al, 1989). Although it has been suggested that the initiation of angiogenesis occurs simultaneous to invasion (reviewed by Bergers \& Benjamin, 2003), only one previous study has investigated angiogenesis at the individual stages of the hyperplasia, in situ and invasive breast carcinoma spectrum (Pavlakis et al, 2008). We show that angiogenesis is significantly increased early in this sequence, confirming this recent data, although the greatest increase occurs with tumour invasion, with associated increases in HIF-1 $\alpha$, VEGF and TF expression. Our data therefore suggest that angiogenesis is initiated at the start of hyperplasia, with further increases between in situ and invasive carcinomas. Angiogenesis thus starts with hyperplasia before there is any morphological evidence of atypia.

Endoglin has been reported to be highly expressed in the vasculature of various tumour types, including invasive breast carcinomas (Wang et al, 1994; Beresford et al, 2006), and predicts poor survival and poor clinical response to chemotherapy (Charpin-Taranger et al, 2003; Dales et al, 2004; Beresford et al, 2006). To our knowledge, endoglin has not previously been assessed in each stage of the hyperplasia, in situ and invasive
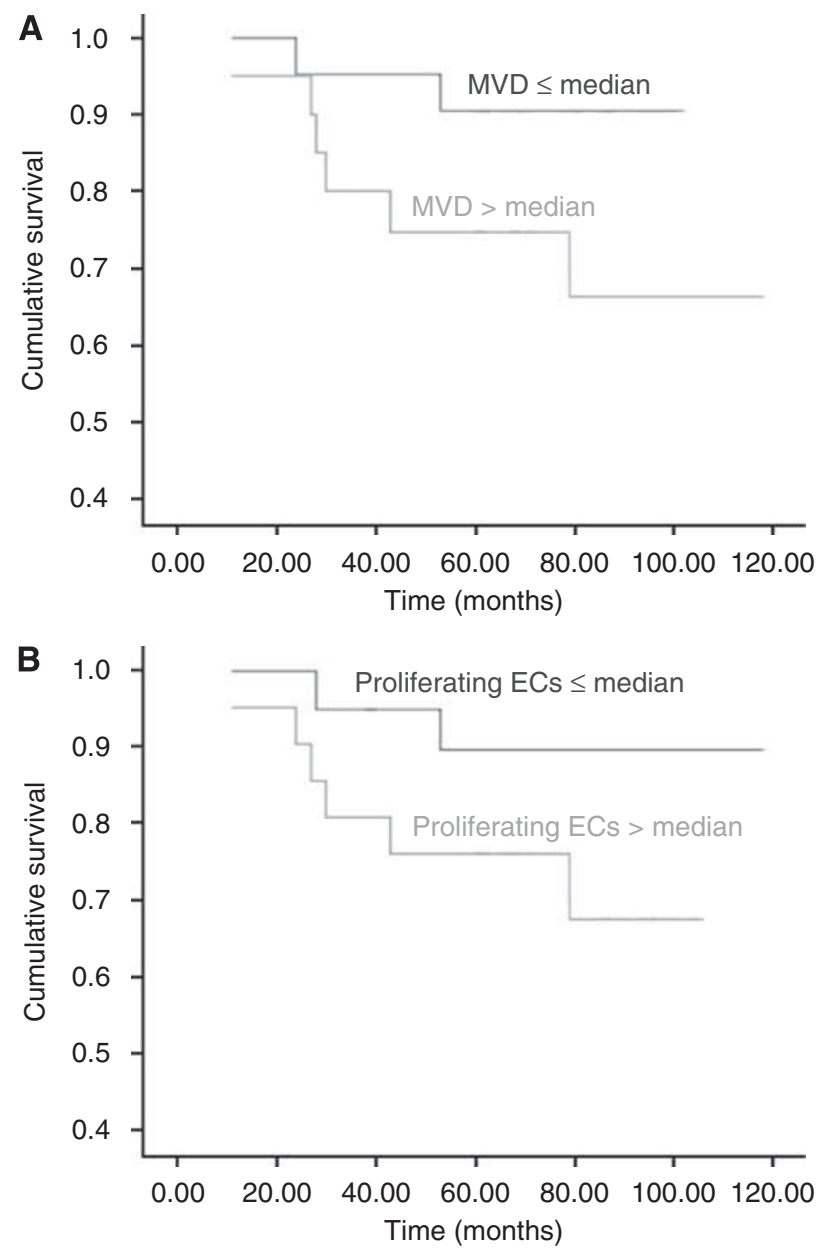

Figure 4 Cox regression survival graphs. Comparison of overall survival between groups of patients with $\leqslant$ median and $>$ median cumulative Chalkley scores (CCS) for $(\mathbf{A})$ MVD $(C D 31 ; P=0.09)$ and $(\mathbf{B})$ proliferating ECs (endoglin; $P=0.12$ ).

breast carcinoma sequence. The significant increase in the percentage of specimens expressing endoglin in both high-grade in situ and invasive carcinomas indicates increases in actively proliferating vessels participating in angiogenesis. In agreement with other studies (El-Gohary et al, 2007), we identified a significant correlation between vessels stained with endoglin and CD31. However, in this study, between one and two-thirds of the vessels within pre-malignant, preinvasive and invasive breast cancers exhibited proliferating ECs (based on CCS calculated with CD31 and endoglin staining) in contrast to a report by Wang et al (1994), which showed that endoglin stained only $20 \%$ of the vessels highlighted by CD31 in invasive breast carcinomas.

Previous studies have showed that a high MVD significantly predicts poor survival of breast cancer patients (both relapse-free survival and overall survival) (Uzzan et al, 2004) as does a high expression of endoglin (Charpin-Taranger et al, 2003; Dales et al, 2004). However, in this study, although patients with a high MVD $(>59)$ and high number of proliferating ECs $(>22)$ tended to have a poorer prognosis than patients with a low MVD $(\leqslant 59)$ and low number of proliferating ECs $(\leqslant 22)$ to the same order of magnitude as reported by the previous studies, these did not achieve significance. This may be because of the relatively small number of invasive cancer patients in this study, which was not primarily designed to address this issue.

In addition, a positive correlation was observed between HIF- $1 \alpha$ and MVD, and between HIF- $1 \alpha$ and proliferating ECs (based on 
endoglin expression). Endoglin expression associated with hypoxia within solid tumours has been reported to be directly due to HIF- $1 \alpha$ binding to the hypoxia response element in the endoglin promoter (Bos et al, 2005). As expected, we showed a significant association between HIF- $1 \alpha$ and VEGF in both ECs and epithelial/ tumour cells, as it is known that the HIF-1 complex recognises a consensus hypoxia response element in the promoter of VEGF that mediates hypoxic signals including angiogenesis. Much less expected was the high percentage of hyperplastic breast lesions that expressed HIF- $1 \alpha$, as a recent investigation reported that HIF$1 \alpha$ was not expressed in hyperplastic lesions (Hao et al, 2007). Although hypoxia is one of the most potent stimulators of VEGF, interestingly, not all of the in situ and invasive cancers expressed HIF- $1 \alpha$. Accumulating evidence indicates that HIF-1-independent pathways can also control angiogenesis (Mizukami et al, 2004). Knockdown of HIF- $1 \alpha$ through small interfering RNA in a colon cancer cell xenograft reduced tumour growth, but surprisingly did not inhibit tumour angiogenesis (Mizukami et al, 2005). The specific molecular mechanisms that underlie HIF-1-independent regulation of VEGF are only now being elucidated, but the RAS oncogene seems to have a pivotal role (Ryan et al, 2000; Mizukami et al, 2004).

Although VEGF is a key mediator of angiogenesis in breast cancer and it is well recognised that VEGF is upregulated in invasive breast carcinomas (Borgstrom et al, 1999), much less is known about VEGF expression in hyperplastic and preinvasive lesions. Indeed, although VEGF expression has recently been reported in hyperplastic epithelium (Pavlakis et al, 2008), this study is the first to show that this is an upregulation compared with normal breast tissue. The constitutive expression of VEGF in normal breast endothelium may be related to the role of VEGF in preventing apoptosis, which promotes EC survival (Gerber et al, 1998), although it is not yet clear how the breast vasculature remains quiescent in the presence of VEGF. Vascular endothelial growth factor-A expression has previously been shown to be significantly associated with MVD in breast cancer (Bolat et al, 2006; Pavlakis et al, 2008). Moreover, the positive correlations seen in this study between VEGF expression and both MVD and proliferating ECs in hyperplasia, in situ and invasive breast carcinoma spectrum indicates that VEGF has a key role in breast cancer angiogenesis, most likely by binding to VEGF receptors on ECs and inducing EC proliferation (Ferrara et al, 2003).

The upregulation of TF in invasive breast cancers may be related to the role of TF in metastasis (Bromberg et al, 2001; Amarzguioui et al, 2006; Ngo et al, 2007). Tissue factor is highly expressed in metastatic, but not in non-metastatic breast carcinoma cells (Bluff et al, 2006), and may contribute to the metastatic process directly through the TF-FVIIa complex and/or through downstream generation of active coagulation factors (Booden et al, 2004; Palumbo et al, 2007). Furthermore, TF-FVIIa signalling via PAR-2 has been shown to stimulate breast cancer cell migration (Jiang et al, 2004; Morris et al, 2006), and a humanised anti-TF monoclonal antibody (CNTO 859) inhibited experimental in vivo lung metastasis from invasive breast cancer cells by more than $99 \%$, indicating a role for TF in breast cancer cell metastasis (Ngo et al, 2007).

The cytoplasmic domain of TF seems to be essential for the production of VEGF in human gastric cancer (Zhang et al, 2005) and melanoma (Abe et al, 1999) cells. A positive feedback mechanism induces upregulation of VEGF on tumour cells, further increasing TF expression (Zhang et al, 1994). In addition, VEGF has also been reported to induce TF expression in ECs, possibly through early growth response gene-1 and sp1 transcription factors (Armesilla et al, 1999; Mechtcheriakova et al, 1999). In agreement with these studies, both TF and VEGF were expressed by vessels at the earliest stages of dysplasia and by ECs associated with in situ cancers in this study. However, interestingly, both VEGF and TF were rarely expressed by vessels within invasive carcinomas. This may be because of the fact that the plethora of angiogenic molecules increases with malignant progression (reviewed by Folkman, 2007), rendering the tumour less dependent on EC-derived VEGF or TF. Our data suggest that, although VEGF and TF are significant factors in promoting angiogenesis, other molecules, for example, fibroblast growth factor-2 and plateletderived growth factor, may also have a significant role after downregulation of VEGF and TF within intratumoural vessels (upon tumour invasion).

This is the first report, to our knowledge, that significantly correlates TF expression with MVD and proliferating ECs in the hyperplasia, in situ and invasive breast carcinoma spectrum. The association between VEGF and TF in ECs suggests a role for both factors in tumour angiogenesis. Furthermore, the association between VEGF and TF in invasive tumour cells, potentially indicates an intimate relationship in breast cancer disease progression, as has been previously shown (Ueno et al, 2000). In addition, macrophages are a major population of infiltrating cells in the tumour stroma; it is possible that VEGF and TF-expressing macrophages, observed in this study, may have a role in regulating the angiogenic switch and tumour progression in human breast cancer, as previously described in an autochthonous mouse model of breast cancer (Lin et al, 2006).

In conclusion, this is the first study to assess angiogenesis, proliferating ECs, HIF- $1 \alpha$, VEGF and TF in each stage of the hyperplasia, in situ and invasive breast carcinoma sequence in a cohort of patients. These data suggest that the angiogenic switch occurs at the onset of hyperplasia in the mammary milk duct before any morphological evidence of atypia, although the greatest increase in angiogenesis occurs between in situ and invasive disease. Hypoxia-inducible factor- $1 \alpha$, VEGF and TF expression are significantly associated with increased angiogenesis and proliferating ECs. Moreover, a significant association between VEGF and TF in both ECs and invasive tumour cells suggests the importance of both these factors in breast cancer angiogenesis, disease progression and tumour biology. Further work is needed to examine the precise relationship between VEGF and TF in breast carcinogenesis, and to establish whether TF has a key independent role in tumour angiogenesis and disease progression that is functionally distinct from the role in VEGF production.

\section{ACKNOWLEDGEMENTS}

The support of Yorkshire Cancer Research is gratefully acknowledged.

\section{REFERENCES}

Abe K, Shoji M, Chen J, Bierhaus A, Danave I, Micko C, Casper K, Dillehay DL, Nawroth PP, Rickles FR (1999) Regulation of vascular endothelial growth factor production and angiogenesis by the cytoplasmic tail of tissue factor. Proc Natl Acad Sci USA 96: 8663-8668

Amarzguioui M, Peng Q, Wiiger MT, Vasovic V, Babaie E, Holen T, Nesland JM, Prydz H (2006) Ex vivo and in vivo delivery of anti-tissue

factor short interfering RNA inhibits mouse pulmonary metastasis of B16 melanoma cells. Clin Cancer Res 12: 4055 - 4061

Armesilla AL, Lorenzo E, Gómez de Arco P, Martinez-Martinez S, Alfranca A, Redondo JM (1999) Vascular endothelial growth factor activates nuclear factor of activated $\mathrm{T}$ cells in human endothelial cells: a role for tissue factor gene expression. Mol-Cell Biol 19: 2032-2043 
Beresford MJ, Harris AL, Ah-See M, Daley F, Padhani AR, Makris A (2006) The relationship of the neo-angiogenic marker, endoglin, with response to neoadjuvant chemotherapy in breast cancer. Br J Cancer 95: $1683-1688$

Bergers G, Benjamin LE (2003) Tumorigenesis and the angiogenic switch. Nat Rev Cancer 3: $401-410$

Bluff JE, Brown NJ, Reed MWR, Staton CA (2008) Tissue factor angiogenesis and tumour progression. Breast Cancer Res 10: 204-214

Bluff JE, Mangera A, Brown NJ, Reed MWR, Staton CA (2006) Tissue factor and vascular endothelial growth factor in breast cancer. Angiogenesis 9: P31

Bolat F, Kayaselcuk F, Nursal TZ, Yagmurdur MC, Bal N, Demirhan B (2006) Microvessel density, VEGF expression, and tumor-associated macrophages in breast tumors: correlations with prognostic parameters. J Exp Clin Cancer Res 25: 365-372

Booden MA, Eckert LB, Der CJ, Trejo J (2004) Persistent signaling by dysregulated thrombin receptor trafficking promotes breast carcinoma cell invasion. Mol Cell Biol 24: 1990-1999

Borgstrom P, Gold DP, Hillan KJ, Ferrara N (1999) Importance of VEGF for breast cancer angiogenesis in vivo: implications from intravital microscopy of combination treatments with an anti-VEGF neutralizing monoclonal antibody and doxorubicin. Anticancer Res 19: $4203-4214$

Bos R, van Diest PJ, de Jong JS, van der Groep P, van der Valk P, van der Wall E (2005) Hypoxia-inducible factor-1alpha is associated with angiogenesis, and expression of bFGF, PDGF-BB, and EGFR in invasive breast cancer. Histopathology 46: 31-36

Bromberg ME, Bailly MA, Konigsberg WH (2001) Role of proteaseactivated receptor 1 in tumor metastasis promoted by tissue factor. Thromb Haemost 86: 1210 - 1214

Charpin-Taranger C, Dales JP, Garcia S, Andrac-Meyer L, Ramuz O, Carpentier-Meunier S, Bonnier P (2003) The immunohistochemical expression of CD105 is a marker for high metastatic risk and worse prognosis in breast cancers. Bull Acad Natl Med 187: 1129-1145, discussion 1145-1146

Chen WT, Huang CJ, Wu MT, Yang SF, Su YC, Chai CY (2005) Hypoxiainducible factor-1alpha is associated with risk of aggressive behavior and tumor angiogenesis in gastrointestinal stromal tumor. Jpn J Clin Oncol 35: $207-213$

Dales JP, Garcia S, Andrac L, Carpentier S, Ramuz O, Lavaut MN, Allasia C, Bonnier P, Charpin C (2004) Prognostic significance of angiogenesis evaluated by CD105 expression compared to CD31 in 905 breast carcinomas: correlation with long-term patient outcome. Int J Oncol 24: $1197-1204$

El-Gohary YM, Silverman JF, Olson PR, Liu YL, Cohen JK, Miller R, Saad RS (2007) Endoglin (CD105) and vascular endothelial growth factor as prognostic markers in prostatic adenocarcinoma. Am J Clin Pathol 127: $572-579$

Ferlay J, Autier P, Boniol M, Heanue M, Colombet M, Boyle P (2007) Estimates of the cancer incidence and mortality in Europe in 2006. Ann Oncol 18: $581-592$

Ferrara N, Gerber HP, LeCouter J (2003) The biology of VEGF and its receptors. Nat Med 9: 669-676

Folkman J, Watson K, Ingber D, Hanahan D (1989) Induction of angiogenesis during the transition from hyperplasia to neoplasia. Nature 339: $58-61$

Folkman J (1995) Angiogenesis in cancer, vascular, rheumatoid and other disease. Nat Med 1: 27-31

Folkman J (2007) Angiogenesis: an organizing principle for drug discovery? Nat Rev Drug Discov 6: 273-286

Gerber HP, McMurtrey A, Kowalski J, Yan M, Keyt BA, Dixit V, Ferrara N (1998) Vascular endothelial growth factor regulates endothelial cell survival through the phosphatidylinositol $3^{\prime}$-kinase/Akt signal transduction pathway. Requirement for Flk-1/KDR activation. J Biol Chem 273: 30336-30343

Hao LS, Wang G, Qian K, Luo T, Li XJ, Wu XT (2007) HIF-1alpha expression and relationship involving tumour cell proliferation and angiogenesis in human breast carcinoma. Sichuan Da Xue Xue Bao Yi Xue Ban 38: $60-63$

Jiang X, Bailly MA, Panetti TS, Cappello M, Konigsberg WH, Bromberg ME (2004) Formation of tissue factor-factor VIIa-factor Xa complex promotes cellular signaling and migration of human breast cancer cells. J Thromb Haemost 2: 93-101

Jones LJ (2006) Progression of ductal carcinoma in situ: the pathological perspective. Breast Cancer Res 8: 204

Lin EY, Li JF, Gnatovskiy L, Deng Y, Zhu L, Grzesik DA, Qian H, Xue XN, Pollard JW (2006) Macrophages regulate the angiogenic switch in a mouse model of breast cancer. Cancer Res 66: 11238-11246

Mechtcheriakova D, Wlachos A, Holzmuller H, Binder BR, Hofer E (1999) Vascular endothelial cell growth factor-induced tissue factor expression in endothelial cells is mediated by EGR-1. Blood 93: 3811-3823

Mizukami Y, Li J, Zhang X, Zimmer MA, Iliopoulos O, Chung DC (2004) Hypoxia-inducible factor-1-independent regulation of vascular endothelial growth factor by hypoxia in colon cancer. Cancer Res 64: 1765-1772

Mizukami Y, Jo WS, Duerr EM, Gala M, Li J, Zhang X, Zimmer MA, Iliopoulos O, Zukerberg LR, Kohgo Y, Lynch MP, Rueda BR, Chung DC (2005) Induction of interleukin-8 preserves the angiogenic response in HIF-1alpha-deficient colon cancer cells. Nat Med 11: 992-997

Mizukami Y, Kohgo Y, Chung DC (2007) Hypoxia inducible factor-1 independent pathways in tumor angiogenesis. Clin Cancer Res 13: 5670 - 5674

Molnar S, Guglielmone H, Lavarda M, Rizzi ML, Jarchum G (2007) Procoagulant factors in patients with cancer. Hematology 12: 555-559

Morris DR, Ding Y, Ricks TK, Gullapalli A, Wolfe BL, Trejo J (2006) Protease-activated receptor-2 is essential for factor VIIa and Xa-induced signaling, migration, and invasion of breast cancer cells. Cancer Res 66: $307-314$

Nakasaki T, Wada H, Shigemori C, Miki C, Gabazza EC, Nobori T, Nakamura S, Shiku H (2002) Expression of tissue factor and vascular endothelial growth factor is associated with angiogenesis in colorectal cancer. Am J Hematol 69: 247-254

Ngo CV, Picha K, McCabe F, Millar H, Tawadros R, Tam SH, Nakada MT, Anderson GM (2007) CNTO 859, a humanized anti-tissue factor monoclonal antibody, is a potent inhibitor of breast cancer metastasis and tumor growth in xenograft models. Int J Cancer 120: 1261-1267

Palumbo JS, Talmage KE, Massari JV, La Jeunesse CM, Flick MJ, Kombrinck KW, Hu Z, Barney KA, Degen JL (2007) Tumor cellassociated tissue factor and circulating hemostatic factors cooperate to increase metastatic potential through natural killer cell-dependent andindependent mechanisms. Blood 110: 133-141

Pavlakis K, Messini I, Vrekoussis T, Yiannou P, Keramopoullos D, Louvrou N, Liakakos T, Stathopoulos EN (2008) The assessment of angiogenesis and fibroblastic stromagenesis in hyperplastic and pre-invasive breast lesions. BMC Cancer 8: 88

Rak J, Milsom C, May L, Klement P, Yu J (2006) Tissue factor in cancer and angiogenesis: the molecular link between genetic tumor progression, tumor neovascularization, and cancer coagulopathy. Semin Thromb Hemost 32: $54-70$

Ryan HE, Poloni M, McNulty W, Elson D, Gassmann M, Arbeit JM, Johnson RS (2000) Hypoxia-inducible factor-1alpha is a positive factor in solid tumor growth. Cancer Res 60: 4010-4015

Ueno T, Toi M, Koike M, Nakamura S, Tominaga T (2000) Tissue factor expression in breast cancer tissues: its correlation with prognosis and plasma concentration. $\mathrm{Br} J$ Cancer 83: 164-170

Uzzan B, Nicolas P, Cucherat M, Perret GY (2004) Microvessel density as a prognostic factor in women with breast cancer: a systematic review of the literature and meta-analysis. Cancer Res 64: 2941-2955

Vermeulen PB, Gasparini G, Fox SB, Colpaert C, Marson LP, Gion M, Belien JA, de Waal RM, Van Marck E, Magnani E, Weidner N, Harris AL, Dirix LY (2002) Second international consensus on the methodology and criteria of evaluation of angiogenesis quantification in solid human tumours. Eur J Cancer 38: 1564-1579

Wang JM, Kumar S, Pye D, Haboubi N, al-Nakib L (1994) Breast carcinoma: comparative study of tumor vasculature using two endothelial cell markers. J Natl Cancer Inst 86: 386-388

Wiechmann L, Kuerer HM (2008) The molecular journey from ductal carcinoma in situ to invasive breast cancer. Cancer 112: 2130-2142

Zhang Y, Deng Y, Luther T, Muller M, Ziegler R, Waldherr R, Stern DM, Nawroth PP (1994) Tissue factor controls the balance of angiogenic and antiangiogenic properties of tumor cells in mice. J Clin Invest 94: 1320 - 1327

Zhang J, Ding J, Zhang X, Shao X, Hao Z (2005) Regulation of vascular endothelial growth factor (VEGF) production and angiogenesis by tissue Factor (TF) in SGC-7901 gastric cancer cells. Cancer Biol Ther 4: 769-772 